\title{
5. Umgang mit Ärger und Frust in der Psychiatrie, oder: Wie bleibe ich cool?
}

\author{
Eva Weiß
}

Häufig fragt man sich als Berufsanfänger, ob das Gefühl von Ärger und Frust überhaupt zulässig ist, wenn man einen helfenden Berufgewählt hat.

Eindeutig ja! Sie haben ein Recht, sich zu ärgern. Sie haben ebenso das Recht, sich zu freuen, sich zu ängstigen, ratlos zu sein, sich ausgeliefert und ohnmächtig zu fühlen, zuversichtlich zu sein, $z u$ hoffen. Sie haben ein Recht auf die gesamte Gefühlspalette eines Menschen.

Der Anspruch an sich selbst, alles verstehen zu müssen, immer über den Dingen $\mathrm{zu}$ stehen, allwissend und gelassen zu sein, ist überhöht, unrealistisch und letztlich wenig hilfreich.

Sie haben ein Recht auf Ihre Gefühle, denn Sie sind auch ein Mensch.

Aber: In Ihrem Job, und ganz speziell in der Psychiatrie, müssen Sie lernen, mit Ihren Gefühlen professionell umzugehen. Sie müssen sie steuern können. Um effektiv zu therapieren, ist eine vertrauensvolle und gute Atmosphäre zu schaffen. Da psychiatrische Patienten beim Beziehungsaufbau oft große Probleme haben und Kommunikationsstörungen, Misstrauen und weitere Faktoren zur Symptomatik gehören, ist es an Ihnen, eine gute Beziehung und Gesamtatmosphäre zu erreichen.

Zum positiven Beziehungsaufbau ist ein professioneller Umgang mit Ihren Gefühlen eine Grundvoraussetzung bei der Arbeit in der Psychiatrie. 
„Ich bin so cool und ärgere mich über so gut wie gar nichts, da stehe ich doch drüber.“

Schön, wenn es so ist.

Psychiatrische Patienten haben oft ein sehr feines Gespür für den Anderen, auch wenn es erst einmal gar nicht so wirkt. Sie spüren manchmal schneller, was mit Ihnen los ist, ehe Sie es selbst merken.

An Quellen für Ärger wie auch für Freude mangelt es im Klinikalltag nicht.

Sie müssen in verschiedenen Ebenen der sozialen Kommunikation rasch switchen. Eine Ebene sind Ihre Vorgesetzten, Kollegen und Teammitglieder. Eine weitere ist die Verwaltungsebene. Die dritte Ebene sind Ihre Patienten und deren Angehörige.

Auf allen Ebenen treten häufig soziale Konflikte auf. Sie können in fünf Untergruppen eingeteilt werden:

\section{Beurteilungskonflikte}

- In der Visite beklagt sich die Patientin bei Ihrem Oberarzt, dass es ihr immer noch nicht besser gehe, obwohl sie schon so lange da sei.

- Sie selbst sind jedoch froh darüber, dass ihr Denken klarer geworden ist, ihr Schlaf schon gebessert ist und nach Ihrer Einschätzung die Therapie anschlägt.

Beurteilungskonflikte entstehen häufig aus unterschiedlichem Vorwissen, mangelhafter Information und unterschiedlichen Einstellungen.

\section{Zielkonflikte}

- Angehörige erwarten eine vollständige Genesung in kürzester Zeit und nicht „nur“ Linderung wesentlicher Symptome.

- Ihr Therapievorschlag wird erst widerspruchslos von Ihrem Patienten hingenommen, aber bei dessen Umsetzung treten offene oder verdeckte Widerstände auf, weil er sich eigentlich von einem anderen Medikament mehr verspricht.

- Ihre Patientin hat andere Mitpatienten mehr im Blick und setzt sich für sie ein, als sich um ihre eigenen Probleme zu kümmern. Alltagsthemen der Station, wie z.B. Sauberkeit und Essen, geraten (überwertig) in den Mittelpunkt der Therapie.

Zielkonflikte entstehen aus gegensätzlichen Zielsetzungen und konkurrierenden Absichten.

\section{Rollenkonflikte}

- Sie erfahren, dass hinter Ihrem Rücken abschätzig über Sie geredet wird.

- Der Ehemann traut Ihrem Wissen nicht und kommt mit „besseren“ Diagnosen und Behandlungsvorschlägen, z.B. aus dem Internet.

- Die Pflegekräfte tragen Beschwerden der Patienten, Besucher oder Angehörigen vor. Dabei ist zu spüren, dass sie selbst auch so denken. 
- Ihr Chef fragt an, was es mit den Beschwerden von Patient X auf sich hat. Sie haben noch nichts davon gehört und konnten dadurch nichts klären.

Rollenkonflikte entstehen durch Differenzen zwischen dem erwarteten Rollenverhalten und wie Sie die Rolle ausfüllen.

\section{Verteilungskonflikte}

- Ihr Patient hat oft, trotz Ihrer knappen zeitlichen Ressourcen, mehr Gesprächskontakte erhalten, als geplant. Nun fordert er dringlich einen erneuten ungeplanten Gesprächstermin.

- Sie sind für viel mehr und schwierigere Patienten zuständig als Ihr Kollege.

- Ihr Oberarzt nimmt sich kaum Zeit für Sie und Ihre Patienten.

Verteilungskonflikte entstehen häufig wegen knapper Ressourcen, ungerechter Verteilung und mangelnder Zuwendung.

\section{Beziehungskonflikte}

- Der Patient will sich von Ihnen nicht behandeln lassen. Er spricht die sympathische Kollegin an und möchte zu ihr wechseln.

- Angehörige lehnen Ihre Gesprächsvorschläge ab, weil sie von der Psychiatrie nichts halten. „Da setze ich keinen Fuß rein, die sind ja alle bekloppt.“

Beziehungskonflikte entstehen häufig durch Antipathien. Sie sind abhängig von Persönlichkeitsstrukturen und unterschiedlichen Wert-, Verhaltens-, Wahrnehmungs- und Deutungsmustern. Auch Vorurteile und Intoleranz sind dafür wichtige Faktoren.

Was können Sie bei Ärger sofort machen?

- Wenn Sie spüren, dass Ärger und Wut in Ihnen hochkommt, dann ,stop‘. Halten Sie inne, bis Ihnen eine vernünftige Reaktion eingefallen ist.

Was könnte eine vernünftige Reaktion sein?

- Grundsätzlich ist es hilfreich, das Gefühl wahrzunehmen und die Kontrolle darüber zu bekommen.

- Ablenkung jeglicher Art ist effektiv, um eine Eskalation zu verhindern und Ihr Gefühl zum Abklingen zu bringen.

- Ist Ihr Ärger sehr groß, sollten Sie sich z.B. ein Glas kaltes Wasser holen und tief durchatmen.

- Bewegen Sie sich. Wenn das nicht geht, weil Sie z.B. nicht aus der Visite gehen können, ist es gut, zu schweigen, um nichts Unbedachtes zu sagen. Bringen Sie sich innerlich zur Ruhe und überdenken die Situation.

- Um Missverständnissen als häufiger Quelle von Streit vorzubeugen, hören Sie aufmerksam zu und fragen Sie notfalls noch einmal nach oder lassen es sich mit den Worten des Patienten wiedergeben. Merkblätter nützen. 
- Wenn Sie jemand offensichtlich ärgern oder provozieren will, ist es gut, das geflissentlich zu überhören.

Lenken Sie sich ab, bewegen Sie sich, wenn Sie können, versuchen Sie herauszubekommen, was das für ein Gefühl ist und geben Sie inm nicht impulsiv nach.

Was sollten Sie nicht machen? Was ist eine unvernünftige Reaktion?

- Dem verständlichen Impuls des Heimzahlenwollens und der Rache nachzugeben.

Wie würde Rache aussehen?

- Sich direkt zu rächen, hieße, den Patienten zurechtzuweisen, ihn anzuschreien, Ihre Macht auszuspielen und ihn z.B. stehen zu lassen, eine sofortige Ausgangssperre zu verhängen, Gesprächswünsche abzuwehren, Medikamente anbzw. abzusetzen, geringschätzig und abwertend über oder mit ihm zu reden, mit dem Oberarzt zu „drohen“, seine Verlegung oder Entlassung zu veranlassen.

- Indirekte Rache äußerte sich, indem Sie Termine nicht einhielten, kurz angebunden wären, mit Fachsprache ihm Ihre Überlegenheit demonstrierten, Informationen vorenthielten.

Vermeiden Sie bei innerer Erregung spontane Äußerungen und Handlungen, es könnte schädlich sein.

Was sollten Sie bei psychiatrischen Patienten und deren Angehörigen speziell beachten?

- Sie treffen auf Menschen mit psychischen Störungen. Was heißt das? Ihre Wahrnehmung, ihr Denken, Fühlen, Verhalten bzw. ihre Erlebnisverarbeitung und ihre sozialen Beziehungen sind krankhaft beeinträchtigt. Diese Störungen sind der willentlichen Steuerung durch den Patienten nicht mehr oder nur zum Teil zugänglich.

- In der Regel kennen Sie die Patienten nicht in gesunden Zeiten. Der Kontakt entsteht erst im Krankheitsfall.

- Deshalb können Ihnen Veränderungen nicht so ins Auge springen. Sie müssen sie explorieren und im Verlauf systematisch beobachten.

Daraus entsteht die wesentliche Frage: Was werten Sie als Krankheitssymptom und wie reagieren Sie adäquat darauf?

Patienten mit einem Abhängigkeitssyndrom haben das unabweisbare Verlangen, sich in einen bestimmten Erlebniszustand zu versetzen. Diesem Verlangen werden die Kräfte des Verstandes untergeordnet. Es beeinträchtigt die freie Entfaltung einer Persönlichkeit und zerstört die sozialen Bindungen und die sozialen Chancen eines Individuums.

Welche Situationen lösen bei diesem Störungsbild häufig Ärger aus? 
- Ihr Patient kommt verspätet und betrunken oder im schlechtesten Fall gar nicht aus dem Urlaub zurück.

- Ihr Patient verlässt die Station und bricht die Therapie vorzeitig ab.

- Ihr Patient trinkt Alkohol. Er betreibt Substanzmissbrauch auf Station.

- Sie erfahren, dass Ihr Patient sturzbetrunken und in schlechtem Zustand in der Notaufnahme war. Er war vor kurzer Zeit von Ihnen stabilisiert entlassen worden.

Das Verlangen nach der Substanz (Craving) ist nicht mit nur einer Entgiftung dauerhaft unter Kontrolle zu bekommen. Wenn dem so wäre, hätten Sie falsch diagnostiziert.

Die affektiven Störungen sind vor allem durch eine klinisch bedeutsame, willentlich nicht kontrollierbare Veränderung der Stimmungslage gekennzeichnet. Die Grundstimmung kann in Richtung Depression gedrückt oder in Richtung Manie gesteigert sein. Stationär sind am häufigsten Patienten mit Depressionen.

Für die Diagnose werden Antrieb, Spontaneität, vegetative Funktionen (Schlafbedürfnis, Appetit, Libido) und soziale Interaktionen mitbetrachtet. Im Erleben der Betroffenen ist die Stimmung sehr gedrückt und es können irreale Befürchtungen vorliegen.

Welche Situationen lösen bei diesem Störungsbild häufig Ärger aus?

- Ihre Patientin fragt nach, ob Sie denn wirklich in Ihrem jugendlichen Alter ein „richtiger“ Arzt seien und nicht nur ein Praktikant.

- Ihre Patientin spricht immer wieder zum selben Thema und dreht sich scheinbar im Kreis.

- Obwohl Sie ihr wiederholt Hoffnung gemacht haben, sieht sie weiterhin alles grau in grau.

- Ihr Patient zieht sich immer auf sein Bett zurück, obwohl ihm Aktivitäten gut tun würden.

- Laut Bericht der Nachtschwestern hat der Patient geschlafen, er klagt selbst aber über durchwachte Nächte.

- Ihre Patientin beharrt auf ihrer Meinung, eine Demenz zu haben und zu verarmen, was objektiv nicht stimmt.

- Sie erfahren zufällig von schweren Ehekonflikten, die bei Ihrer Exploration verneint worden waren.

- Die Genesung stellt sich nur zögerlich ein. Es wird von den Angehörigen eine Verlegung in ein anderes Krankenhaus gefordert.

Bei Patienten mit Depressionen ist Ihr therapeutischer Optimismus gefragt, auch wenn Ihre Patienten die Fortschritte erst später erkennen können.

Bei gerontopsychiatrischen Patienten treffen Sie am häufigsten auf Demenzen.

Eine Demenz führt zum Verlust kognitiver emotionaler und sozialer Fähigkeiten, was zur Beeinträchtigung sozialer Funktionen führt. Es gehen besonders bereits erworbene Denkfähigkeiten verloren. Weiterhin können das Kurzzeitgedächtnis, 
die Sprache und die Motorik und bei einigen Formen auch die Persönlichkeit betroffen sein.

Wie spüren Sie Ihren Ärger darüber im Stationsalltag?

- „Das habe ich Ihnen doch schon gestern erklärt, hören Sie nicht richtig zu?“

- "Ich habe nicht so viel Zeit, antworten Sie mir auf die einfache Frage.“

- „Sie müssen mir das schon genauer erklären, damit ich Sie verstehen kann.“

- Die Patientin will immer wieder mit zur Stationstür hinaus, obwohl sie nicht orientiert ist.

\section{Die Gehirnerkrankung verlangsamt das Tempo, setzt Grenzen und erzeugt nerven-} de Redundanzen.

Als Psychose bezeichnet man eine schwere psychische Erkrankung, die mit einem zeitweiligen weitgehenden Verlust des Realitätsbezugs und starker sozialer Beeinträchtigung einhergeht.

Welche Situationen lösen bei diesem Störungsbild häufig Ärger aus?

- Ihr Patient verhält sich unerwartet und sonderbar. Sein Verhalten entbehrt jeder Logik.

- Sie müssen ihn explorieren, aber er sagt nichts. Sie haben das Gefühl, dass Sie „Luft“ für ihn sind.

- Sie spüren eine Feindseligkeit Ihnen gegenüber, die durch nichts begründet scheint.

- Ihr Patient bricht abrupt den guten Gesprächskontakt ab und starrt an die Decke.

- Ihr Patient lässt sich nicht dazu bewegen, aus dem Bett aufzustehen, geschweige denn, sich zu duschen.

- Ihre Patientin lehnt die verordnete Medikation ab, spuckt sie aus oder versteckt sie in den Pflanzen.

- Achtung: Mütter von psychotischen Patienten können zu Recht sehr allergisch auf (vermutete) Schuldzuweisungen reagieren.

Das Verhalten von Patienten mit akuten Psychosen ist oft nicht vorhersehbar. Im Sozialkontakt sind sie geringer belastbar und schnell durch Reizüberflutung überfordert. Lassen Sie sich nicht irritieren und bewahren Sie die Ruhe. Machen Sie lieber kurze und häufigere Interventionen.

Das Verhalten von Persönlichkeitsstörungen weicht in charakteristischer Weise von einem flexiblen, situationsangemessenen Erleben und Verhalten ab. Es ist unflexibel oder wenig angepasst.

Welche Situationen lösen häufig Ärger, aber auch Ängste aus? Zum Beispiel Drohungen:

- Nach einem Suizidversuch besteht Ihre Patientin auf ihrer sofortigen Entlassung und droht mit einem erneuten Suizidversuch, wenn das nicht geschieht.

- Sie droht indirekt, wie „wenn Sie das tun, dann wissen Sie, was passiert“. 
- Es wird mit der Öffentlichkeit gedroht, z.B. „das bringe ich in die Presse“.

- Es wird angedroht, „zum Chef zu gehen“.

Nehmen Sie Drohungen bei Persönlichkeitsstörungen immer ernst, geben Sie ihnen aber nie nach. Nehmen Sie den Druck heraus durch einen Zeitaufschub. Da Sie als Anfänger noch nicht die volle alleinige Entscheidungsbefugnis für das prinzipielle therapeutische Vorgehen haben, müssen Sie sich sich ohnehin noch das Okay von Ihrem Oberarzt einholen.

- Vereinbarte Regeln werden gebrochen.

- Die anfängliche Therapiemotivation schwindet, ist nicht mehr zu erkennen.

Bei Patienten mit Persönlichkeitsstörungen ist ein respektierendes, konsequentes, aber nicht starres Verhalten Ihrerseits gefordert.

Bei traumatisierten Patientinnen und Patienten treten nach einem traumatischen Ereignis unterschiedliche psychische und psychosomatische Symptome auf. Häufig kommt es zu einem Gefühl von Hilflosigkeit sowie zu einer Erschütterung des Selbst- und Weltverständnisses durch das traumatische Erleben.

Welche Situationen lösen häufig Ärger und Ratlosigkeit aus?

- Kurz vor der geplanten Entlassung teilt die Patientin Ihnen mit, dass sie nicht in ein traumatisierendes Umfeld zurückkehren kann.

- Die Patientin nimmt nicht am üblichen Stationsleben teil und zieht sich ohne erkennbaren Grund zurück. Aktivierungsversuche Ihrerseits laufen ins Leere.

- Bei anamnestischen Erhebungen berichtet sie nur sehr zögerlich und mauert bei einigen Fragen.

- Ihre Patientin lehnt vehement eine notwendige körperliche Untersuchung ab.

- Ihre Patientin sieht keine Möglichkeit, wie ihr zu helfen sei. Es sei alles zwecklos.

Traumatisierte Patienten sprechen, wenn überhaupt, erst sehr spät und unter Druck über diese Tatsache. Sie müssen diese Hypothese im Kopf haben und behutsam abklären.

Welche Ärger-Kontrollstrategien können Sie anwenden?

- Analysieren Sie und denken Sie um. Wenn Sie Ärger aufsteigen fühlen, treten Sie innerlich einen Schritt zur Seite und fragen Sie sich, was Sie auf die Palme treibt. Kann es sein, dass Sie sich gerade darüber ärgern wollen, dass Ihr Patient psychisch krank ist? Dass ein Patient mit einem gebrochenen Bein nicht gut laufen kann, ärgert Sie doch auch nicht. Ihr Fachwissen kann Ihnen bei der Kontrolle Ihres Gefühls helfen.

- Achten Sie auf sich. Lassen Sie Fragen, Stress und negative Gefühle in der Klinik. Nutzen Sie Freizeit zur Erholung. Ausgeruht sind Sie ausgeglichener. Das tut allen gut. 
- Holen Sie sich Hilfe. Sie arbeiten zwar eigenständig, aber im Team und unter fachlicher Anleitung. Sie müssen also nicht alle Probleme allein lösen. Es ist nichts Ehrenrühriges daran, sich zu beraten oder in schwierige Situationen nicht allein zu gehen. Sprechen Sie mit Ihrem Oberarzt darüber. Nutzen Sie auch das Angebot von Supervisionen.

- Zeitmanagement. Legen Sie fest, wie lange Sie Zeit eingeplant haben und sagen Sie das zu Beginn des Kontakts und halten Sie es ein.

- Streben Sie kleine und realistische Therapieziele an. So halten Sie Enttäuschungen in Grenzen.

- Seien Sie geduldig. Widerstehen Sie dem verständlichen Drang, das Leiden rasch $\mathrm{zu}$ beenden oder wenigstens zu lindern. Machen Sie sich klar, dass Sie Menschen mit überwiegend chronischen Syndromen und mit Komorbiditäten behandeln. Das braucht einfach Zeit.

- Lachen Sie drüber. Lachen baut Stress ab. Aber beachten Sie, wo Sie lachen. Unsichere Mithörer könnten sich „verlacht“ fühlen.

- Lenken Sie sich ab von kreisenden Gedanken. Wenn etwas schiefgegangen ist, lassen Sie keine Befürchtungen aufkommen, dass Sie nun nicht mehr akzeptiert werden. Jeder macht Fehler. Lenken Sie Ihre Aufmerksamkeit konsequent auf ein erfreulicheres Thema.

- Suchen Sie nicht ausschließlich die Schuld bei sich. Berufsanfänger neigen dazu, ihren eigenen Einfluss zu überschätzen und sich Misserfolge anzulasten.

- Vielleicht haben Sie Glück im Unglück? Es hätte noch schlimmer kommen können. Sie erfahren etwas, was sonst nicht sichtbar geworden wäre.

Worauf sollten Sie langfristig achten?

- Wenn Sie sich sehr oft ärgern, ist das nicht gut für Sie und ein wichtiges Achtungssignal.

- Ärger und Frust werden häufig dadurch ausgelöst, dass Sie sich unsicher, ängstlich oder ohnmächtig erleben, sich angegriffen, nicht ernst genommen oder missachtet fühlen. Ihr Selbstwertgefühl ist verletzt.

- Als Berufsanfänger können Sie die Souveränität der erfahrenen Kollegen natürlich noch nicht haben. Geduld und Zuversicht kommen mit der Zeit.

- Wenn sich die Sicherheit und Gelassenheit nicht einstellen wollen und der Ärger rasch da ist, beobachten Sie sich genauer: Auf was „springen“ Sie besonders an? Was nervt und ärgert Sie konkret?

- Es liegen Antipathien vor. Ist Ihnen Ihr Gegenüber unsympathisch?

- Sind Sie zu anspruchsvoll, zu perfektionistisch?

- Zweifeln Sie an Ihrer Berufswahl?

- Sind Sie grundsätzlich leicht reizbar?

- Sind Sie selbstunsicher? Haben Sie eine schlechte Phase z.B. wegen persönlicher Schwierigkeiten?

- Wenn es immer dieselben Situationen sind, die Sie sauer werden lassen, haben Sie mehrere Möglichkeiten, darauf zu reagieren: 
- Weitermachen wie bisher, sich weiterärgern und darauf vertrauen, dass es Ihnen bald besser geht. Gefühle haben keinen Dauerbestand und klingen ja von allein ab.

- Reagieren Sie einmal anders als üblich und beobachten Sie mit Neugier, was passiert.

- Machen Sie sich Beobachtungen aus dem Team zunutze. Andere sehen durch den Abstand deutlicher, woran es klemmt.

- Sprechen Sie mit Vertrauenspersonen darüber. Ihr Oberarzt hat sicher auch ein offenes Ohr.

- Wenn sich dadurch nichts bessert, die Anderen bei ständiger Wiederholung nur die Augen verdrehen, holen Sie sich professionelle Hilfe wie z.B. in der Balintoder IFA-Gruppe oder in der Selbsterfahrung.

Unsicherheit, Anspannung und Ärgernisse sind beim Start in den Job normal. Wenn Sie mehr Frust spüren als Zufriedenheit, Stolz, Freude, Anerkennung und Gelassenheit, dann holen Sie sich professionelle Hilfe.

„Das Ärgerliche am Ärger ist, dass man sich schadet, ohne anderen zu nützen.“

- Kurt Tucholsky

\section{Literaturempfehlung}

Bonacker T (2008) Sozialwissenschaftliche Konflikttheorien. Eine Einführung. 4. Aufl. Verlag für Sozialwissenschaften Wiesbaden

Mager K (2002) Bevor Sie aus der Haut fahren - Wie Sie fair und selbstbewusst Konflikte meistern. 2. Aufl. Rowohlt Reinbek bei Hamburg

Yalom ID (2010) Theorie und Praxis der Gruppentherapie. Ein Lehrbuch. Leben Lernen 66. Klett-Cotta Stuttgart 